\title{
CSF Biomarkers for Early Diagnosis of Synucleinopathies: Focus on Idiopathic RBD
}

\author{
Claudio Liguori ${ }^{1}$ - Federico Paolini Paoletti ${ }^{2}$. Fabio Placidi ${ }^{1} \cdot$ Roberta Ruffini $^{1}$ • Giulia Maria Sancesario ${ }^{3}$. \\ Paolo Eusebi $^{2} \cdot$ Nicola Biagio Mercuri $^{3,4} \cdot$ Lucilla Parnetti $^{2}$
}

Published online: 14 January 2019

(C) Springer Science+Business Media, LLC, part of Springer Nature 2019

\begin{abstract}
Purpose of Review Idiopathic REM sleep behavior disorder (iRBD) is one of the most significant prodromal manifestations of synucleinopathies. Different predictive biomarkers for iRBD conversion have been investigated, but scarce data are present in literature about the predictive role of cerebrospinal fluid (CSF) biomarkers. In this review, we focus on CSF biomarkers in patients with both iRBD and RBD associated with synucleinopathies to explore their potential predictive power.

Recent Findings Recent studies revealed that CSF $\alpha$-synuclein levels are higher in Parkinson's disease (PD) patients with RBD compared to those without RBD, even if $\alpha$-synuclein does not seem to predict conversion of iRBD into PD. In the Parkinson Progression Marker Initiative (PPMI) cohort, early PD patients with RBD show lower CSF A $\beta_{42}$ levels, which predict faster cognitive decline. CSF prion protein and inflammatory biomarkers have been also investigated in RBD and synucleinopathies with controversial results.

Summary A variety of CSF biomarkers are promising candidate for predicting iRBD conversion into synucleinopathies. Further studies are needed in iRBD patients followed for several years in order to observe the phenoconversion in synucleinopathies and to elucidate the possible role of CSF biomarkers as predictive biomarkers of conversion.
\end{abstract}

Keywords Idiopathic RBD · CSF · Biomarkers · Synucleinopathy · Neurodegeneration

\section{Introduction}

Rapid eye movement (REM) sleep behavior disorder (RBD) is a sleep disorder featured by enacting dreams associated with the loss of muscle atonia during REM sleep. When occurring

Claudio Liguori and Federico Paolini Paoletti contributed equally to this work.

This article is part of the Topical Collection on Sleep

Claudio Liguori

dott.claudioliguori@yahoo.it

1 Sleep Medicine Centre, Department of Systems Medicine, University of Rome 'Tor Vergata', Viale Oxford, 8100133 Rome, Italy

2 Section of Neurology, Department of Medicine, University of Perugia, Perugia, Italy

3 IRCCS Fondazione Santa Lucia, Rome, Italy

4 Neurology Unit, Department of Systems Medicine, University of Rome "Tor Vergata", Rome, Italy isolated, it has been defined as idiopathic RBD (iRBD), a condition in which RBD is not associated with other neurologic disorders; on the other hand, symptomatic (or secondary) RBD indicates the presence of RBD associated with other neurologic disturbances such as narcolepsy or neurodegenerative diseases, and in particular synucleinopathies. However, it is now well ascertained that $\mathrm{RBD}$ represents a potential harbinger of neurodegenerative disorders, in particular synucleinopathies including Parkinson's disease (PD), multisystem atrophy (MSA), and dementia with Lewy bodies (DLBs). The association between RBD and synucleinopathies is mainly due to the impairment of specific brainstem areas, especially mesencephalon and pontine tegmentum, which are involved in the pathogenesis of both conditions [1]. Although the diagnosis of PD is centered on cardinal motor signs, $\alpha$ synuclein-pathology starts several years before the occurrence of motor symptoms. Hence, the concept of prodromal or premotor stages of $\mathrm{PD}$ have been recently introduced to define the stages in which patients present early clear non-motor symptoms but subtle motor signs, which are not sufficient to meet the current diagnostic criteria for PD [2]. Converging 
evidence indicates that $\mathrm{RBBD}$ is the most specific prodromal symptom of $\alpha$-synucleinopathies; the great majority of patients initially diagnosed as iRBD eventually develop a Lewy body disorder during the following years [3]. The prodromal phase of $\alpha$-synucleinopathies is of great interest, relating to the possible development of disease-modifying and neuroprotective therapies, which should be introduced as earliest as possible. Accordingly, validation of specific markers for prodromal illness represents a key challenge and several efforts have been made to identify biomarkers predicting the conversion of iRBD into synucleinopathy. Hence, in this review, we will put our attention on CSF biomarkers in RBD, highlighting their potential ability to predict conversion of iRBD in synucleinopathies.

\section{REM Sleep Behavior Disorder}

RBD belongs to sleep parasomnias, which are defined as sleep disorders characterized by undesirable physical events or experiences occurring in association with sleep (at sleep onset, during sleep, or after arousal from sleep). Parasomnias are divided in two main groups represented by non-REM (NREM) sleep parasomnias (including sleepwalking, sleep terrors, and confusional arousals) and REM sleep parasomnias; among them, RBD is the most representative. It consists of loss of muscular atonia during REM sleep (the so-called REM sleep without atonia, RSWA), associated with a history of nocturnal distressing dreaming and complex behavior [4]. Although a series of specific questionnaires have been validated to detect RBD among general population [5], its diagnosis relies on video-polysomnography (vPSG). According to the 3rd Edition of International Classification of Sleep Disorders [6], diagnosis of RBD requires the presence of RSWA on nocturnal vPSG, in association with sleep-related injurious (or potentially injurious) abnormal and vigorous nocturnal complex motor behaviors and vocalizations, either derived by history or documented during a single night of vPSG [6]. iRBD is considered a rare disease and its prevalence has been estimated between 0.38 and $0.5 \%$ in large populationbased studies [7]. Moreover, it is a male predominant disorder, usually diagnosed between 50 and 85 years old [8].

The circuitry involved in RBD is not fully understood. However, different brain areas including the primary and premotor cortices with their input from basal ganglia, brainstem, and spinal cord motor generators seem to be involved [9]. Briefly, two motor systems, present in the region of midbrain and pons, are implicated in REM sleep muscle atonia: the sublaterdorsal nucleus in the "REM-on" region (it actives two inhibitory pathways, the "direct route" and the "indirect route," both inhibiting the motor neurons) and the locomotor generators, not yet identified in humans, responsible for suppressing locomotor activity. These brain regions appeared to be dysregulated in animal models of RSWA and thus can underline the clinical disorder [9].

\section{RBD as Prodromal Feature of Synucleinopathies}

Evidence deriving from different cohort studies indicates that iRBD is one of the most relevant predictor of synucleinopathies. Shenck and co-authors [3] showed that a very high percentage (about $81 \%$ ) of patients initially diagnosed as iRBD eventually developed a parkinsonism/dementia syndrome, mainly PD and DLB, and less frequently MSA. The risk of developing synucleinopathy increases along the follow-up, with a rate of conversion ranging from $33 \%$ after a 5-year follow-up to $91 \%$ after a 14-year follow-up [10]. However, it has been reported that time interval between iRBD and subsequent neurodegenerative disorder can range up to 50 years, suggesting that $\alpha$-synuclein pathology starts several decades before the appearance of the first subtle symptoms of PD, MSA, and DLB [11]. Findings suggest that iRBD patients, even when remaining clinically free from synucleinopathies, show the decreased striatal dopamine transporter (DAT) uptake on [123]I-FP-CIT SPECT or the appearance of substantia nigra (SN) hyperechogenicity on transcranial sonography during the follow-up, suggesting the future development of synucleinopathy [12].

Nevertheless, it should be mentioned that only a quarter of PD patients manifests RBD before the occurrence of parkinsonism, another quarter tends to have a simultaneous onset of RBD and parkinsonism, and the remaining 50\% develops REM parasomnia once motor disturbances have already manifested [13]. A possible explanation for this heterogeneity is that RBD is associated with specific motor phenotype, and it has been found to occur earlier and more frequently in patients with postural instability/gait difficulty phenotype [14].

As reported by a recent meta-analysis [15], the prevalence of RBD has been estimated up to $88 \%$ in MSA and more than half of patients reported symptoms of RBD before the onset of motor deficits. In DLB, RBD is a part of core clinical features since it has been discovered to improve the diagnostic accuracy of autopsy-confirmed DLB. Moreover, RBD starts several years before the occurrence of other symptoms and tends to become less vigorous or even quiescent over time [16].

\section{Predictive Biomarkers for Conversion of iRBD in Synucleinopathies: State of the Art}

Patients with iRBD can develop a variety of other early nonmotor symptoms, which do not fulfill the current diagnostic criteria for synucleinopathies but suggest that the underlying neurodegenerative process has already started. iRBDassociated non-motor symptoms include olfactory loss, 
autonomic dysfunction, impaired color vision, constipation, depression, and cognitive impairment. Longitudinal studies revealed that, among them, impaired olfaction and impaired color vision are the most powerful predictor for conversion of iRBD into synucleinopathies [17, 18••, 19].

Imaging of nigro-striatal dopaminergic pathway can provide information about RBD conversion. On $99 \mathrm{mTc}-$ TRODAT-1 SPECT, iRBD patients presenting lower DAT density in the putamen have shorter conversion-free survival time [20••]. Meanwhile, on [123]I-FP-CIT, a DAT deficit greater than $25 \%$ in the putamen is able to define iRBD patients with higher risk to develop synucleinopathies along a 3year follow-up (see Table 1) [21••].

SN hyperecogenicity on transcranial sonography, if used in association with [123]I-FP-CIT, increases the sensibility of detecting iRBD patients converting in synucleinopathies up to $100 \%$, whereas the sensitivity of baseline SN hyperechogenicity alone is near $43 \%$ (see Table 1) [22].

Studies based on electroencephalography revealed that iRBD patients developing a neurodegenerative disorder present greater delta and theta frequencies compared to patients with iRBD and synucleinopathy-free [23••].

Magnetic resonance imaging (MRI) techniques, especially resting-state functional MRI and 7-T susceptibility-weighted imaging, have been studied, but, to date, it does not seem to play a specific role for predicting phenoconversion of patients with iRBD [24, 25].

\section{CSF Biomarkers in Synucleinopathies: an Overview}

It is now well accepted that CSF, strictly in contact with neurons, is a source of biomarkers potentially offering the most promising insight into the pathogenesis of neurodegenerative disorders since their early stages. CSF has been extensively investigated in terms of both diagnostic and prognostic value in synucleinopathies, considering several biomarkers.

$\alpha$-Synuclein is a cytosolic protein widely expressed in brain and mostly localized in presynaptic neuronal terminals where it

Table 1 Clinical and imaging predictors of $\mathrm{R} B \mathrm{BD}$ conversion

Clinical signs:

Impaired olfactory function

Impaired color vision

Nigro-striatal imaging:

Putamen:

- Marked dopamine transporter (DAT) deficit ([123]I-FP-CIT)

- Reduced DAT density (99mTc-TRODAT-1 SPECT)

Substantia nigra:

- Hyperechogenicity (transcranial sonography) acts as a mediator of neurotransmitters release, vesicle trafficking, and synaptic plasticity [25]. It is the major component of Lewy bodies, the most representative pathological hallmark of synucleinopathies. After its discovery in the CSF, the role of $\alpha$ synuclein as a biomarker of synucleinopathies has been largely investigated. Different studies revealed that CSF total $\alpha$ synuclein ( $\mathrm{t}-\alpha$-syn) levels are decreased in synucleinopathies compared to other neurodegenerative disorders and to healthy subjects, maybe reflecting higher brain levels of pathological deposition of $\alpha$-synuclein in Lewy bodies, similar to pathological "protein trapping" reported for $\beta$-amyloid ${ }_{42}\left(A \beta_{42}\right)$ in Alzheimer's disease (AD) [26-30]. Furthermore, it was found also that both CSF oligomeric $\alpha$-synuclein (o- $\alpha$-syn) levels and o- $\alpha$-syn $/ \mathrm{t}-\alpha$-syn ratio are higher in PD patients compared to healthy controls [30,31]. A similar trend was observed for phosphorylated $\alpha$-synuclein ( $\mathrm{p}-\alpha$-syn), showing higher CSF levels in PD patients compared to healthy controls [32]. Postmortem CSF $\mathrm{p}$ - $\alpha$-syn was found significantly raised in MSA, distinguishing it from other synucleinopathies [33]. When CSF $\alpha$-synuclein levels were studied in relation to clinical features, lower CSF $\mathrm{t}-\alpha$-syn and higher $\mathrm{o}-\alpha$-syn levels were detected in the more advanced stages of PD [34, 35].

The coexistence of amyloid plaques and neurofibrillary tangles with Lewy bodies in synucleinopathies [36] is one of the main reasons that have led researchers to investigate core $\mathrm{AD}$ biomarkers (total tau, t-tau; phosphorylated tau, $p$-tau; and $A \beta_{42}$ ) even in the CSF of patients affected by synucleinopathies. Several studies highlighted the prognostic value of $A \beta_{42}$ in terms of development of cognitive impairment: lower CSF levels strongly predict greater and faster cognitive decline in PD patients [37-39]. Although the controversial results, a similar association has been found for $\mathrm{t}$-tau and $\mathrm{p}$-tau, showing higher CSF levels in PD demented patients compared to non-demented patients [40 ${ }^{\circ}$. Furthermore, $A \beta_{42} / t$-tau ratio improved the diagnostic accuracy of PD patients from controls [30].

In the last few years, besides $\alpha$-synuclein and core $\mathrm{AD}$ biomarkers, different CSF biomarkers have been investigated in synucleinopathies, often with uneven and variable results. They include markers related to genetic forms of PD (i.e., DJ1 and GCase activity) [30, 41], markers of inflammation [42], and markers of neuronal injury other than protein tau such as neurofilament light chain (NfL) [43, 44•].

In Table 2, the results of CSF biomarker studies in PD patients with RBD compared to those without RBD are briefly reported.

\section{CSF Biomarkers in RBD}

\section{a-Synuclein}

In the last years, researchers have compared CSF levels of biomarkers in PD patients with and without RBD, often showing inconclusive and controversial results. 
Table 2 CSF biomarkers in PD patients with RBD compared to those without RBD

\begin{tabular}{|c|c|c|c|}
\hline CSF biomarkers & Study & Findings in PD patients & Correlations in RBD \\
\hline \multirow[t]{3}{*}{$\alpha$-Synuclein species } & Compta et al. (2015) [40•] & $\begin{array}{l}\text { Higher o- } \alpha \text {-syn in NDPD and PDD } \\
\text { vs iRBD and CTRL }\end{array}$ & \\
\hline & Hu et al. (2015) [45•] & Higher o- $\alpha$-syn in RBD vs non-RBD & $\begin{array}{l}\text { RBDSQ scores with higher } \\
\text { o- } \alpha \text {-syn levels }\end{array}$ \\
\hline & Dolatshahi et al. (2018) [46] & $\begin{array}{l}\text { Higher total } \alpha \text {-syn after } 1 \text {-year follow-up } \\
\text { in RBD vs non-RBD }\end{array}$ & $\begin{array}{l}\text { RBDSQ scores with increase of } \\
\alpha \text {-syn levels along time }\end{array}$ \\
\hline \multirow[t]{2}{*}{$\begin{array}{l}\text { Core AD biomarkers } \\
\qquad\left(\mathrm{A} \beta_{42}, \mathrm{t} \text {-tau, } \mathrm{p} \text {-tau }\right)\end{array}$} & Pagano et al. (2018) [47•] & $\begin{array}{l}\text { Lower } A \beta_{42} \text { and higher } t \text {-tau } / A \beta_{42} \\
\text { ratio in RBD vs non-RBD }\end{array}$ & \\
\hline & Ba et al. (2018) [48•] & Lower $A \beta_{42}$ in RBD vs non-RBD & $\begin{array}{l}\text { Lower } A \beta_{42} \text { predicts } \\
\text { cognitive decline }\end{array}$ \\
\hline Prion protein $(\mathrm{PrP})$ & Zhang et al. (2017) [51] & Higher PrP in RBD vs non-RBD & \\
\hline $\begin{array}{l}\text { Inflammatory biomarkers } \\
\text { (NO, IL-1 } \beta, \text { TNF1) }\end{array}$ & Hu et al. (2015) [45•] & Higher NO and IL- $1 \beta$ in RBD vs non-RBD & $\begin{array}{l}\text { RBDSQ with NO and } \\
\text { IL-1 } 1 \beta \text { o- } \alpha \text {-syn with NO, IL- } 1 \beta, \text { TNF1 }\end{array}$ \\
\hline
\end{tabular}

$A \beta_{42} \beta$-amyloid ${ }_{42}, I L-1 \beta$ interleukin- $1 \beta, N O$ nitric oxide, $o$ - $\alpha$-syn oligomeric $\alpha$-synuclein, $R B D S Q$ REM Behavior Disorders Screening Questionnaire, TNF1 tumor necrosis factor $1, t$-tau total tau protein)

$\mathrm{Hu}$ and collaborators [45•], by using the REM Sleep Behavior Disorders Screening Questionnaire (RBDSQ), divided a cohort of PD subjects in patients with and without RBD and found that patients with RBD showed higher CSF and serum levels of o- $\alpha$-syn compared to patients without RBD. They found also that RBDSQ score increased concomitantly with higher CSF o- $\alpha$-syn levels. Accordingly, Dolatshahi and colleagues [46] recruited 112 non-demented PD patients from the Parkinson Progressive Marker Initiative (PPMI), a 5-year observational, multi-center, and international study encompassing 400 recently diagnosed drug-naïve PD patients. They identified greater increase of CSF $\alpha$ synuclein levels after 1-year follow-up in patients with RBD compared to those without RBD. Meanwhile, in the Fox Investigation for New Discovery of Biomarkers in Parkinson's Disease (BioFIND), a cross-sectional and observational study assessing clinical features and CSF biomarkers of 115 moderate and advanced PD, CSF $\alpha$ synuclein levels did not correlate with RBD questionnaire total score [49].

Nevertheless, only a very limited number of studies have been performed in patients manifesting RBD as prodromal feature without evidence of motor signs. Compta and colleagues [40 ${ }^{\bullet}$ measured CSF $\alpha$-synuclein levels in healthy controls, iRBD patients, non-demented PD patients (NDPD) and demented PD patients (PDD) with the purpose of reflecting the prodromal-motor-dementia continuum of PD. Whereas both total $\alpha$-synuclein and oligomeric/ total ratio did not significantly differ among groups, o- $\alpha$ syn showed higher levels in both PD and PDD patients compared to iRBD patients and controls. However, the lack of differences between iRBD and controls did not allow to consider $\alpha$-synuclein, a prodromal marker of PD in patients with RBD.

\section{CSF AD Biomarkers}

Core AD CSF biomarkers have been studied in RBD in a lesser degree compared to $\alpha$-synuclein. The great majority of the available studies do not present significant associations between RBD and CSF levels of AD core biomarkers [46, 49].

However, more recently, Pagano and collaborators measured CSF biomarkers in a large group of drug-naïve early PD patients, showing that patients with RBD had lower CSF $A \beta_{42}$ and greater t-tau/A $\beta_{42}$ ratio compared to patients without RBD [47•]. Interestingly, they found that the severity of RBD correlated with higher amyloid pathology as proved by lower CSF $A \beta_{42}$ concentrations. Furthermore, their findings revealed also that severity of RBD at baseline was predictive for faster cognitive decline in PD patients presenting both greater amyloid and greater $\alpha$-synuclein pathologies. Accordingly, in the PPMI cohort CSF A $\beta_{42}$ levels were significantly lower at baseline in early PD patients with RBD. After 3 years, PD patients with RBD had greater cognitive decline in Montreal Cognitive Assessment (MoCA) score, and lower baseline $\mathrm{A} \beta_{42}$ levels were able to predict cognitive decline only in PD subjects with RBD over the 3-year followup $[48 \cdot]$.

\section{Prion Protein (PrP)}

Mainly expressed in neurons and microglia, prion protein $(\mathrm{PrP})$ is a membrane-binding glycoprotein which is involved in molecular pathways contributing to neuronal differentiation and synaptic development, to cellular oxidative stress response, and to cell-cell adhesion. Some evidence indicates that CSF PrP levels are altered in different neurodegenerative disorders [50]. To date, however, few studies assessing CSF PrP in patients presenting synucleinopathies and RBD have been carried out. Particularly, Zhang and collaborators [51] 
showed that CSF PrP levels were increased in PD patients with RBD compared to those without RBD, suggesting therefore an accelerated neuronal degeneration in RBD.

\section{Inflammatory Markers}

In their works, $\mathrm{Hu}$ and colleagues [45•] found that CSF nitric oxide (NO), CSF interleukin $1 \beta$ (IL-1 $\beta$ ), and serum prostanglandin E2 (PGE2) levels were higher in PD patients with RBD compared to patients without RBD. Moreover, RBDSQ score increased concomitantly with higher levels of these inflammatory biomarkers. They showed also that CSF o- $\alpha$-syn levels significantly correlated with CSF NO, IL- $1 \beta$, and necrosis tumor factor 1 (TNF1) levels in patients with RBD, suggesting a condition of increased microglia activation in the presence of elevated o-a-synuclein CSF levels in the pathophysiology of RBD. Accordingly, in a recent casecontrol study based on PET imaging and involving a series of patients with iRBD and without clinical evidence of parkinsonism and cognitive impairment, increased microglial activation was detected in the substantia nigra along with reduced dopaminergic function in the putamen [52••]. Furthermore, an interesting correlation between CSF inflammatory markers, $\alpha$-synuclein, and markers of neuroaxonal injury (i.e., NfL) has been recently evidenced [50]. Taken together, these findings indicate that neurodegeneration and neuroinflammation are two early and complementary aspects, which interact each other in the pathological network leading to synucleinopathies. Hence, besides $\alpha$-synuclein, markers of inflammation and neuroaxonal injury may represent the future direction in the research focusing on serum and CSF biomarkers in patients with RBD as prodromal feature of $\mathrm{PD}$ and other synucleinopathies.

\section{Conclusions}

Different biomarkers have been investigated for their ability of predicting iRBD phenoconversion into synucleinopathies, so far. They include clinical biomarkers, imaging of nigro-striatal dopaminergic pathway, hyperecogenicity of the $\mathrm{SN}$, brain MRI, and electroencephalography.

CSF biomarkers have been explored in patients with synucleinopathies, especially PD, manifesting RBD or not. In PD, significant differences have been evidenced for $\alpha$-synuclein, core $\mathrm{AD}$ biomarkers, prion protein, and inflammatory biomarkers between patients with RBD and those without RBD. However, the role of CSF biomarkers as predictor of iRBD conversion remains to be defined. The validation of specific CSF biomarkers able to predict the development of synucleinopathies in patients with iRBD is still an unmet need. Based on Lewy body pathology, $\alpha$-synuclein seems the most promising biomarker for predicting the conversion to alphasynucleinopathies in iRBD patients. Moreover, the coexistence in synucleinopathies of amyloid plaques and neurofibrillary tangles with Lewy bodies makes core AD biomarkers interesting as predictive biomarkers of iRBD conversion. Accordingly, CSF A $\beta_{42}$ levels can be used for predicting the cognitive decline in patients affected by iRBD and possibly developing the Lewy body dementia disorders. Meanwhile, the ascertained role of neuroinflammation in neurodegenerative disorders suggests to consider even inflammatory markers and markers of neuronal injury as potential predictive biomarkers.

Further studies should be carried out in patients with iRBD to define the role of CSF biomarkers as predictors of conversion. Longitudinal studies reflecting the prodromal and clinical continuum of synucleinopathies will be extremely useful to elucidate the sensitivity, specificity, reliability, and effect of confounding factors of these biomarkers. Accordingly, CSF biomarkers could gain a prognostic value in patients with iRBD, hopefully predicting the conversion into a specific disease (PD, MSA, or DLB).

\section{Compliance with Ethical Standards}

Conflict of Interest Paolo Eusepi, Claudio Liguori, Nicola Biagio Mercuri, Federico Paolini Paoletti, Lucilla Parnetti, Fabio Placidi, Roberta Ruffini and Giulia Maria Sancesario each declare no potential conflicts of interest.

Human and Animal Rights and Informed Consent This article does not contain any studies with human or animal subjects performed by any of the authors.

Publisher's Note Springer Nature remains neutral with regard to jurisdictional claims inpublished maps and institutional affiliations.

\section{References}

Papers of particular interest, published recently, have been highlighted as:

- Of importance

•- Of major importance

1. Barone DA, Henchcliffe C. Rapid eye movement sleep behavior disorders and link to alpha-synucleinopathies. Clin Neurophysiol. 2018;129:1551-64.

2. Mahlknecht P, Seppi K, Poewe W. The concept of prodromal Parkinson's disease. J Parkinsons Dis. 2015;5(4):681-97.

3. Schenck CH, Boeve BF, Mahowald MW. Delayed emergence of a parkinsonian disorder or dementia in $81 \%$ of older men initially diagnosed with idiopathic rapid eye movement sleep behavior disorder: a 16-year update on a previously reported series. Sleep Med. 2013;14(8):744-8.

4. Schenck C, Bundlie S, Ettinger MG, Mahowald MW. Chronic behavioral disorders of human rem sleep: a new category of parasomnia. Sleep. 1986;9(2):293-308. 
5. Stiasny-Kolster K, Mayer G, Schäfer S, Möller JC, HeinzelGutenbrunner M, Oertel WH. The REM sleep behavior disorder screening questionnaire - a new diagnostic instrument. Mov Disord. 2007 Dec;22(16):2386-93.

6. American Academy of Sleep Medicine. International Classification of Sleep Disorders. $3^{\text {rd }}$ edition Darien. 2014.

7. Ohayon MM, Caulet M, Priest RG. Violent behavior during sleep. J Clin Psychiatry. 1997;58(8):369-76.

8. Peever J, Luppi PH, Montplaisir J. Breakdown in REM sleep circuitry underlies REM sleep behavior disorder. Trends Neurosci. 2014;37(5):279-88.

9. Boeve BF, Silber MH, Ferman TJ, Lin SC, Benarroch EE, Schmeichel AM, et al. Clinicopathologic correlations in 172 cases of rapid eye movement sleep behavior disorder with or without a coexisting neurologic disorder. Sleep Med. 2013;14(8):754-62.

10. Iranzo A, Fernández-Arcos A, Tolosa E, Serradell M, Molinuevo JL, Valldeoriola F, et al. Neurodegenerative disorder risk in idiopathic REM sleep behavior disorder: study in 174 patients. PLoS One. 2014;9(2):e89741.

11. Claassen DO, Josephs KA, Ahlskog JE, Silber MH, TippmannPeikert M, Boeve BF. REM sleep behavior disorder preceding other aspects of synucleinopathies by up to half a century. Neurology. 2010;75(6):494-9.

12. Iranzo A, Tolosa E, Gelpi E, Molinuevo JL, Valldeoriola F, Serradell M, et al. Neurodegenerative disease status and postmortem pathology in idiopathic rapid-eye-movement sleep behaviour disorder: an observational cohort study. Lancet Neurol. 2013;12:443-53.

13. De Cock VC. Restoration of normal motor control in Parkinson's disease during REM sleep. Brain. 2007;130:450-6.

14. Postuma RB. REM sleep behavior disorders in Parkinson's disease is associated with specific motor features. J Neurol Neurosurg Psychiatry. 2008;79:1117-21.

15. Palma JA, Fernandez-Cordon C, Coon EA, Low PA, Miglis MG, Jaradeh S, et al. Prevalence of REM sleep behavior disorder in multiple system atrophy: a multicenter study and meta-analysis. Clin Auton Res Off J Clin Auton Res Soc. 2015;25:69-75.

16. Ferman TJ, Boeve BF, Smith GE, Lin SC, Silber MH, Pedraza O, et al. Inclusion of RBD improves the diagnostic classification of dementia with Lewy bodies. Neurology. 2011;77:875-82.

17. Ferini-Strambi L. Does idiopathic REM sleep behavior disorder (iRBD) really exist? What are the potential markers of neurodegeneration in iRBD? Sleep Med. 2011;12(Suppl 2):S43-9.

18.• Postuma RB, Gagnon JF, Bertrand JA, Génier Marchand D, Montplaisir JY. Parkinson risk in idiopathic REM sleep behavior disorder: preparing for neuroprotective trials. Neurology. $2015 ; 84(11): 1104-13$ This paper documented that the use of simply assessed markers can identify patients with RBD to be included in neuroprotective trials against Parkinson disease, multiple system atrophy, and dementia with Lewy bodies.

19. Rocchi C, Placidi F, Liguori C, Del Bianco C, Lauretti B, Diomedi $\mathrm{M}$, et al. Daytime autonomic activity in idiopathic rapid eye movement sleep behavior disorder: a preliminary study. Sleep Med. 2018;52:163-7.

20.• Li Y, Kang W, Yang Q, Zhang L, Zhang L, Dong F, et al. Predictive markers for early conversion of iRBD to neurodegenerative synucleinopathy diseases. Neurology. 2017;88:1493-500 This research article suggested the predictive value of autonomic dysfunction and DAT uptake in identifying patients with iRBD at a high risk of progressing into neurodegenerative synucleinopathy diseases.

21.• Iranzo A, Santamaria J, Valldeoriola F, Serradell M, Salamero M, Gaig C, et al. Dopamine transporter imaging deficit predicts early transition to synucleinopathy in idiopathic rapid eye movement sleep behavior disorder. Ann Neurol. 2017;82:419-28 This paper quantified the decreased FP-CIT putamen uptake as greater than $25 \%$ able to predict the conversion to synucleinopathy after 3 years' follow-up in idiopathic RBD patients.

22. Rodrigues Brazete J, Gagnon JF, Postuma RB, Bertrand JA, Petit D, Montplaisir J. Electroencephalogram slowing predicts neurodegeneration in rapid eye movement sleep behavior disorder. Neurobiol Aging. 2016;37:74-81.

23.• Rolinski M, Griffanti L, Piccini P, Roussakis AA, SzewczykKrolikowski K, Menke RA, et al. Basal ganglia dysfunction in idiopathic REM sleep behaviour disorder parallels that in early Parkinson's disease. Brain. 2016;139:2224-34 Here Authors proved the very early dysfunction of basal ganglia connectivity in RBD, which was comparable to that of PD, despite obvious differences on dopamine transported single photon emission computerized tomography.

24. Frosini D, Cosottini M, Donatelli G, Costagli M, Biagi L, Pacchetti $\mathrm{C}$, et al. Seven tesla MRI of the substantia nigra in patients with rapid eye movement sleep behavior disorder. Parkinsonism Relat Disord. 2017;43:105-9.

25. Marques O, Outeiro TF. Alpha-synuclein: from secretion to dysfunction and death. Cell Death Dis. 2012;3(7):e350.

26. Shi M, Bradner J, Hancock AM, Chung KA, Quinn JF, Peskind ER, et al. Cerebrospinal fluid biomarkers for Parkinson's disease diagnosis and progression. Ann Neuol. 2011;69:570-80.

27. Parnetti L, Chiasserini D, Bellomo G, Giannandrea D, de Carlo C, Qureshi MM, et al. Cerebrospinal fluid tau/ $\alpha$ synuclein ratio in Parkinson's disease and degenerative dementias. Mov Disord. 2011;26:1428-35.

28. Mollenhauer B, Locascio JJ, Schulz-Schaeffer W, Sixel-Döring F, Trenkwalder C, Schlossmacher MG. $\alpha$-Synuclein and tau concentrations in cerebrospinal fluid of patients presenting with parkinsonism: a cohort study. Lancet Neurol. 2011;10(3):230-40.

29. Parnetti L, Chiasserini D, Persichetti E, Eusebi P, Varghese S, Qureshi MM, et al. Cerebrospinal fluid lysosomal enzymes and alpha-synuclein in Parkinson's disease. Mov Disord. 2014;29(8): 1019-27.

30. Parnetti L, Farotti L, Eusebi P, et al. Differential role of CSF alphasynuclein species, tau, and A $\beta 42$ in Parkinson's disease. Front Aging Neurosci Internet. 2014;6(53).

31. Tokuda T, Qureshi MM, Ardah MT, Varghese S, Shehab SAS, Kasai T, et al. Detection of elevated levels of $\alpha$-synuclein oligomers in CSF from patients with Parkinson's disease. Neurology. 2010;75:1766-70

32. Wang Y, Shi M, Chung KA, et al. Phosphorylated synuclein in Parkinson's disease. Sci Transl Med. 2012;4(121):121ra20 $121 \mathrm{ra} 20$.

33. Foulds PG, Yojota O, Thurston A, Davidson Y, Ahmed Z, Holton J, et al. Post mortem cerebrospinal fluid $\alpha$-synuclein levels are raised in multiple system atrophy and distinguish this from the other $\alpha$ synucleinopathies, Parkinson's disease and dementia with lewy bodies. Neurobiol Dis. 2012;45:188-95.

34. Aasly JO, Johansen KK, Brønstad G, et al. Elevated levels of cerebrospinal fluid $\alpha$-synuclein oligomers in healthy asymptomatic LRRK2 mutation carriers. Front Aging Neurosci. 2014;6:248.

35. Kang J-H, Irwin DJ, Chen-Plotkin AS, et al. Association of cerebrospinal fluid $\beta$-amyloid 1-42, T-tau,P-tau 181, and $\alpha$ synucleinlevelswithclinical features of drug-naive patients with early Parkinson disease. JAMA Neurol Internet 2013;70:1277-1287.

36. Jellinger KA. Neuropathological aspects of Alzheimer disease, Parkinson disease and frontotemporal dementia. Neurodegener Dis. 2008;5(3-4):118-21.

37. Compta Y, Martí MJ, Ibarretxe-Bilbao N, et al. Cerebrospinal tau, phospho-tau, and beta-amyloid and neuropsychological functions in Parkinson's disease. Mov Disord. 2009;24(15):2203-10.

38. Montine TJ, Shi M, Quinn JF, Peskind ER, Craft S, Ginghina C, et al. CSF A $\beta 42$ and tau in Parkinson's disease with cognitive impairment. Mov Disord. 2010;25(15):2682-5. 
39. Leverenz JB, Stennis Watson G, Shofer J, Zabetian CP, Zhang J, Montine TJ. Cerebrospinal fluid biomarkers and cognitive performance in non-demented patients with Parkinson's disease. Parkinsonism Relat Disord. 2011;17(1):61-4.

40. Compta Y, Valente T, Saura J, Segura B, Iranzo Á, Serradell M, et al. Correlates of cerebrospinal fluid levels of oligomeric- and total- $\alpha$-synuclein in premotor, motor and dementia stages of Parkinson's disease. J Neurol. 2015;262(2):294-306 The Authors documented that CSF total- $\alpha$-synuclein levels correlate with CSF tau and $A \beta_{42}$ levels, possibly suggesting that tau pathology may influence the presence of high (instead of low) CSF total- $\alpha$-synuclein levels in the setting of PD-related dementia.

41. Waragai M, Wei J, Fujita M, Nakai M, Ho GJ, Masliah E, et al. Increased level of DJ-1 in the cerebrospinal fluids of sporadic Parkinson's disease. Biochem Biophys Res Commun Internet. 2006;345:967-72.

42. Magdalinou NK, Paterson RW, Schott JM, Fox NC, Mummery C, Blennow $\mathrm{K}$, et al. A panel of nine cerebrospinal fluid biomarkers may identify patients with atypical parkinsonian syndromes. J Neurol Neurosurg Psychiatry. 2015;86(11):1240-7.

43. Bech S, Hjermind LE, Salvesen L, Nielsen JE, Heegaard NH, Jørgensen HL, et al. Amyloid-related biomarkers and axonal damage proteins in parkinsonian syndromes. Parkinsonism Relat Disord. 2012 Jan;18(1):69-72.

44. Hansson O, Janelidze S, Hall S, Magdalinou N, Lees AJ, Andreasson U, et al. Swedish BioFINDER study. Blood-based NfL: a biomarker for differential diagnosis of parkinsonian disorder. Neurology. 2017;88(10):930-7 Authors firstly identified a blood biomarker (NfL) which can be used to distinguish PD from atypical parkinsonisms.

45. Hu Y, Yu SY, Zuo LJ, Cao CJ, Wang F, Chen ZJ, et al. Parkinson disease with REM sleep behavior disorder: features, $\alpha$-synuclein, and inflammation. Neurology. 2015;84(9):888-94 Here, it was documented that elevated $\alpha$-synuclein levels in CSF and serum may be correlated with RBD in PD patients through central and peripheral nervous systems inflammatory mechanisms.

46. Dolatshahi M, Pourmirbabaei S, Kamalian A, Ashraf-Ganjouei A, Yaseri M, Aarabi MH. Longitudinal alterations of alpha-synuclein, amyloid Beta, total, and phosphorylated tau in cerebrospinal fluid and correlations between their changes in Parkinson's disease. Front Neurol. 2018;9:560.

47. Pagano G, De Micco R, Yousaf T, Wilson H, Chandra A, Politis M. REM behavior disorder predicts motor progression and cognitive decline in Parkinson disease. Neurology. 2018;91(10):e894-905 Pagano and co-Authors proved that the presence of RBD in PD patients is associated with faster motor progression when greater synuclein and dopaminergic pathologies are present, and with higher risk of cognitive decline when greater synuclein and amyloid pathologies are present.

48. Ba M, Yu G, Kong M, Liang H, Yu L. CSF A $\beta_{1-42}$ level is associated with cognitive decline in early Parkinson's disease with rapid eye movement sleep behavior disorder. Transl Neurodegener. 2018;7:22 This paper suggested that the addition of CSF $\mathbf{A} \boldsymbol{\beta}_{\mathbf{4 2}}$ assessment to RBD increases the likelihood of identifying early PD patients at high risk for cognitive decline.

49. Goldman JG, Andrews H, Amara A, Naito A, Alcalay RN, Shaw LM, et al. Cerebrospinal fluid, plasma, and saliva in the BioFIND study: relationships among biomarkers and Parkinson's disease features. Mov Disord. 2018;33(2):282-8.

50. Hall S, Janelidze S, Surova Y, Widner H, Zetterberg H, Hansson O. Cerebrospinal fluid concentrations of inflammatory markers in Parkinson's disease and atypical parkinsonian disorders. Sci Rep. 2018;8(1):13276.

51. Zhang WJ, Shang XL, Peng J, Zhou MH, Sun WJ. Expression of prion protein in the cerebrospinal fluid of patients with Parkinson's disease complicated with rapid eye movement sleep behavior disorder. Genet Mol Res. 2017;23:16 (1).

52.• Stokholm MG, Iranzo A, Østergaard K, Serradell M, Otto M, Svendsen KB, et al. Assessment of neuroinflammation in patients with idiopathic rapid-eye-movement sleep behaviour disorder: a case-control study. Lancet Neurol. 2017;16(10):789-96 This original research documented that increased microglial activation (detected by PET scans) in the substantia nigra along with reduced dopaminergic function in the putamen is present in patients affected by idiopathic RBD. 